\title{
A diffuse interface method with real-gas thermodynamic equilibrium closure applied to capillary problems
}

\author{
Thomas Laroche*1, Nicolas Odier ${ }^{1}$, Thomas Schmitt ${ }^{2}$, Milan Pelletier ${ }^{2}$, Bénédicte Cuenot ${ }^{1}$ \\ ${ }^{1}$ Centre Européen de Recherche et de Formation Avancée en Calcul Scientifique, CERFACS \\ - 42 avenue Gaspard Coriolis 31057 Toulouse Cedex 01, France \\ ${ }^{2}$ Laboratoire EM2C, CNRS, CentraleSupélec, Université Paris-Saclay, 3 rue Joliot Curie, \\ 91192, Gif-sur-Yvette Cedex, France \\ ${ }^{*}$ Corresponding author email: laroche@cerfacs.fr
}

\begin{abstract}
Liquid injection and atomization are critical phenomena for two-phase turbulent combustion problems. A diffuse interface method (DIM) is considered in this paper to numerically predict the liquid-gas interface through a diffuse zone of artificially mixed multiple phases, assumed to be at thermodynamic equilibrium. This model relies on a multi-phase equilibrium solver integrated in the finite-element solver AVBP, used to study the liquid-gas equilibrium of various mixtures of practical interest in the recent work of Pelletier et al. [2]. Surface tensions are considered in this work, based on the incompressible formulation of Brackbill [4] and the compressible extension of Perigaud and Saurel [5]. The Laplace test case and a binary collision of liquid droplets are performed. Then a turbulent liquid injection is computed in two dimensions for two Weber numbers. Results indicate that the model exhibits the formation of ligaments at the origin of primary atomization.
\end{abstract}

\section{Keywords}

Diffuse Interface Method, Atomization, Real Gas, Multiphase Flow, Surface tension.

\section{Introduction}

Atomization phenomena are known to be of primarily importance for the resulting combustion quality, and need to be predicted with accuracy. However, their numerical prediction still remains a challenge, due to the high density and velocity gradients that must be numerically resolved, the multi-scale nature of the problem and the high level of complexity of the physics involved. The destabilization of the injected liquid core leads to the development of liquid ligaments, which burst into small droplets, until their evaporation and combustion. In these atomization processes, surface tension is a key parameter (Lefebvre [11]).

Several methodologies have been proposed to numerically predict atomization processes with reasonable accuracy, such as Volume Of Fluid (VOF) (Hirt and Nichols [8]) and the Level Set method (Osher and Sethian [7]). However, in the context of high-pressure combustion experienced in real industrial system, a compressible solver able to handle multi-phase flow must be considered. In this paper, the diffuse interface method (DIM) developed by Pelletier et al. ([2], [16]) within the unstructured and massively parallel solver AVBP (Schonfeld and Rudgyard [12]) is extended to account for capillary effects. Such methods require a two-phase thermodynamic closure to properly compute the mixture properties. At high pressure, a natural choice is to use cubic equation of state, also able to describe real gas effects above the critical point (Poling et al. [9]). Two academic test case are first considered to validate the description of surface tension effects, and a 2D turbulent jet is then computed with capillary effects. Results are discussed to show the model potential and identify further research axes.

\section{Flow Model}

Assuming the equilibrium of pressure, velocity, temperature and chemical potential between the phases, the 3 equations Homogeneous Equilibrium for the Navier-Stokes single-component 
system is given by:

$$
\begin{gathered}
\frac{\partial \rho}{\partial t}+\vec{\nabla} \cdot(\rho \vec{u})=0 \\
\frac{\partial \rho \vec{u}}{\partial t}+\vec{\nabla} \cdot(\rho \vec{u} \otimes \vec{u}+P \underline{\underline{\mathbf{l}}})=\vec{\nabla} \cdot(\underline{\underline{\tau}})+\vec{f}_{v} \\
\left.\frac{\partial \rho e_{t o t}}{\partial t}+\vec{\nabla} \cdot\left(\rho e_{t o t}+P\right) \vec{u}\right)=\vec{\nabla} \cdot(\underline{\underline{\tau}} \cdot \vec{u})+\vec{u} \cdot \vec{f}_{v}
\end{gathered}
$$

where $\rho, \vec{u}$ and $P$ are respectively the mixture density, velocity and pressure. The total energy is $e_{t o t}=e_{c}+e_{s}$, with $e_{c}=u^{2} / 2$ the kinetic energy and $e_{s}$ the sensible energy. $\mu, \tau$ and $f_{v}$ respectively represent the fluid viscosity, the viscous stress tensor and the surface tension volume force which will be detailed in the following sections. The DIM describes the flow as a multi-phase mixture where gaseous and liquid phases are likely to coexist. The mixture density and sensible energy are thus defined as mixture variables:

$$
\begin{gathered}
\rho=\alpha_{l} \rho_{l}+\left(1-\alpha_{l}\right) \rho_{v} \\
\rho e_{s}=\alpha_{l} \rho_{l} e_{s, l}+\left(1-\alpha_{l}\right) \rho_{v} e_{s, v}
\end{gathered}
$$

where $\alpha_{l}$ is the liquid volume fraction. $e_{s, l}$ and $e_{s, v}$ are the sensible energies respectively computed at $\rho_{l}$ and $\rho_{v}$, the liquid and vapor densities at saturation.

The fluid state is assumed to consist in either a single phase (when it is thermodynamically stable), or otherwise in a two-phase mixture equilibrium.

\section{Real Gas Thermodynamic closure}

The thermodynamic closure chosen in the present work is the Peng-Robinson cubic equation of state [9]:

$$
P(V, T, \underline{Y})=\frac{R T}{V-b_{m}}-\frac{a_{m}(T)}{V^{2}+2 b_{m} V-b_{m}^{2}}
$$

where $a_{m}$ and $b_{m}$ are respectively the attractive interaction coefficient and the effective molecular volume of the mixture. For a multicomponent mixture, those two coefficients are computed following the van der Waals mixing rule (Poling et al. [9]) as a function of the local composition. This cubic equation of state is valid in the pure liquid and vapor regions. For a single-component two-phase mixture, the thermodynamic equilibrium is assumed to follow the relations Eqs. 7 to 9

$$
\begin{aligned}
P_{v} & =P_{l} \\
T_{v} & =T_{l} \\
g_{v} & =g_{l}
\end{aligned}
$$

allowing to compute the density and sensible energy for a two-phase mixture following Eqs. 4 and 5. The liquid and vapor densities at saturation $\rho_{l}$ and $\rho_{v}$ in Eq. 4 are computed thanks to a tabulation of the saturation curve as detailed in the work of Pelletier [19].

The liquid/gas composition $\alpha_{l}$ is finally given by a simple extrapolation of density in the unstable, two-phase region of the saturation curve as written in Eq. 4.

\section{Capillary effects}

Among the phenomena responsible for the destabilization of the dense liquid core during atomization, capillary effects induce a surface force applied to the infinitely thin interface depending on the pressure difference between the two phases following Landau and Lifshitz [18]:

$$
\left(P_{l}-P_{v}+\sigma \kappa\right) \vec{n}=\left(\tau_{l}-\tau_{v}\right) \vec{t}+\vec{\nabla}_{s} \sigma
$$


where $P_{\phi}$ is the phase pressure in phase $\phi \in\{l, v\}, \sigma$ the surface tension coefficient, $\kappa$ the interface curvature, $\vec{n}$ and $\vec{t}$ are respectively the normal and tangent to the interface. The RHS of this equation involves the viscous stress tensor $\tau_{\phi}$ for phase $\phi$ as well as the tangential projection of $\vec{\nabla} \sigma$. These two terms are neglected in the approach of Brackbill et al. [4] where the resulting surface force $f_{s}$ writes:

$$
\overrightarrow{f_{s}}=\left(P_{l}-P_{v}\right) \vec{n}=\sigma \kappa \vec{n}
$$

In the diffuse interface formulation, this surface force must be expressed in a volume force form which tends to the surface force as the interface thickness tends to zero. A color function $C$, varying smoothly from 0 to 1 between the two pure phases is used to formulate this force.

$$
\overrightarrow{f_{v}}=\sigma \kappa(\vec{x}) \vec{\nabla} C
$$

This formulation is known as the Continuum Surface Force, or CSF ([4]. The curvature is expressed as the local variation of the interface normal: $\kappa(\vec{x})=\vec{\nabla} \cdot(\vec{\nabla} C /|\vec{\nabla} C|)$. In the present work the liquid volume fraction $\alpha_{l}$ (Eq. 4) is used as color function:

$$
C=\alpha_{l}
$$

The CSF formulation of Brackbill et al. [4] being incompressible, the non-conservative compressible form of Perigaud and Saurel [5] is considered in this work. The final Navier-Stokes system with capillary effects is written in the system of Eqs. 2 and 3.

\section{Validation}

A single-component mixture of $N_{2}$ is considered to investigate the behavior of the surface tension implementation on two test cases.

\section{Laplace pressure equilibrium}

Quantitative validation has been led on the typical test case of the Laplace pressure equilibrium. A round droplet of radius $r_{\text {droplet }}=5 \mu \mathrm{m}$ with isobaric condition at $P=10 \mathrm{Bar}$ is initialized. The surface tension coefficient is $\sigma=0.004134 \mathrm{~N} . \mathrm{m}^{-1}$, while $\rho_{l}=719 \mathrm{~kg} \cdot \mathrm{m}^{-3}$ and $\rho_{v}=45 \mathrm{~kg} \cdot \mathrm{m}^{-3}$ respectively represent the liquid and vapor density. The cartesian grid of 100 cells in each direction allows to resolve the droplet with 30 cells in its radius and 3 cells in the interface thickness. Capillary effects tend to retrieve the pressure difference following Eq. 10, neglecting viscous stress tensor and assuming a constant surface tension coefficient:

$$
P_{l}-P_{v}=\frac{\sigma}{r_{\text {droplet }}}=826.8 \mathrm{~Pa}
$$

In Fig. 1b, the pressure difference between the center and the outside of the droplet is found to adjust the initially constant pressure towards the final jump values. The relative error when the oscillation stops is about $2 \%$. This remaining error on pressure jump is probably due to numerical errors in the estimation of interface curvature on unstructured grids.

Higher order computation of such variable should be therefore considered, and would probably improve the final result. However, the results on the Laplace pressure test remain in the same order of magnitude than the results of Perigaud and Saurel [5].

\section{Validation: Binary collision of droplets.}

A qualitative validation of the surface tension formulation is then achieved through a 3D simulation of the impact of two droplets on a tetrahedral grid of 13 million cells, with 20 cells within the droplet diameter, at constant pressure and temperature. The interface is initialized as a smooth 




(a) Visualization of the pressure field after stabilisation of pressure oscillation.

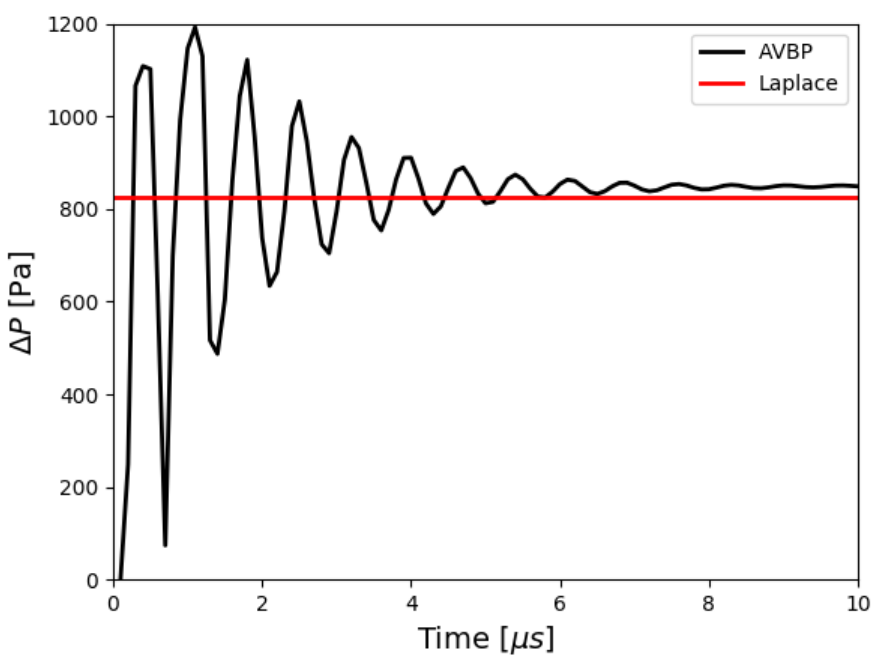

(b) Pressure difference between the inside and outside of the droplet vs time. Results are compared to the theoretical value.

Figure 1. Laplace pressure test case.

transition between the liquid and gaseous saturated states of the fluid, using a hyperbolic tangent in Eq. 15:

$$
\alpha_{l}(x, y, z)=\left(\frac{\rho_{l}+\rho_{v}}{2}\right)+\left(\frac{\rho_{l}-\rho_{v}}{2}\right) \tanh \left(\frac{D / 2-\sqrt{\left(x-x_{0}\right)^{2}+\left(y-y_{0}\right)^{2}+\left(z-z_{0}\right)^{2}}}{2 \epsilon}\right)
$$

with $\rho_{l}=667 \mathrm{~kg} \cdot \mathrm{m}^{-3}$ and $\rho_{v}=32 \mathrm{~kg} \cdot \mathrm{m}^{-3}$ the liquid and vapor density, $\left(x_{0}, y_{0}, z_{0}\right)$ the coordinates of the droplet center and $\epsilon=5 \mu \mathrm{m}$ the thickness of the interface which is resolved with 3 cells. Surface tension effects are characterized with the impact Weber number defined as:

$$
W e_{i m p}=\frac{\rho u_{r}^{2} D}{\sigma}
$$

where $u_{r}=1.56 \mathrm{~m} . \mathrm{s}^{-1}$ is the droplet relative velocity and $D=0.2 \mathrm{~mm}$ their diameter. Two different Weber numbers have been tested: $W e_{i m p}=40$ and $W e_{i m p}=80$. These values allow to study two well-known phenomena of droplet impact: reflective separation with no satellite, and reflective separation with creation of a third droplet (Ashgriz \& Poo [1]). To focus on the effect of the surface tension, the relative velocity of impact is kept the same in all cases.

Figs. $2 \mathrm{a}$ to $2 \mathrm{~d}$ show the results of both cases at four successive times, with an iso-surface $\alpha_{l}=$ 0.5 . Figs. $2 a$ and $2 b$ show the creation of a thick rim during droplet coalescence. In both cases the rim size keeps increasing until the capillary force balances droplet inertia. Fig. $2 c$ shows that this balance is reached earlier in the case $W e=40$ as vertical extension starts earlier than for $W e_{i m p}=80$. Finally, Fig. $2 \mathrm{~d}$ displays two well-known regimes that are expected: reflective separation with no satellite and three-drop reflective separation. Comparing our numerical results to the experimental snapshots in Fig. 3 highlight a shift of a factor two on the recovery of the critical impact Weber number separating the regimes. This could be linked to a difference between the mixture composition considered and further work will be dedicated to understand this issue. However, higher Weber numbers induce higher levels of fragmentation, in agreement with the experimental work of Ashgriz \& Poo [1]. 

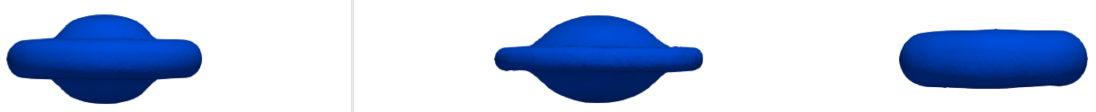

(a) $t=0.31 \mathrm{~ms}$

(b) $t=0.63 \mathrm{~ms}$


(c) $t=1.11 \mathrm{~ms}$

(d) $t=2.56 \mathrm{~ms}$

Figure 2. Time series of droplet binary impact. $W e_{i m p}=40$ left image, $W e i m p=80$ right image.
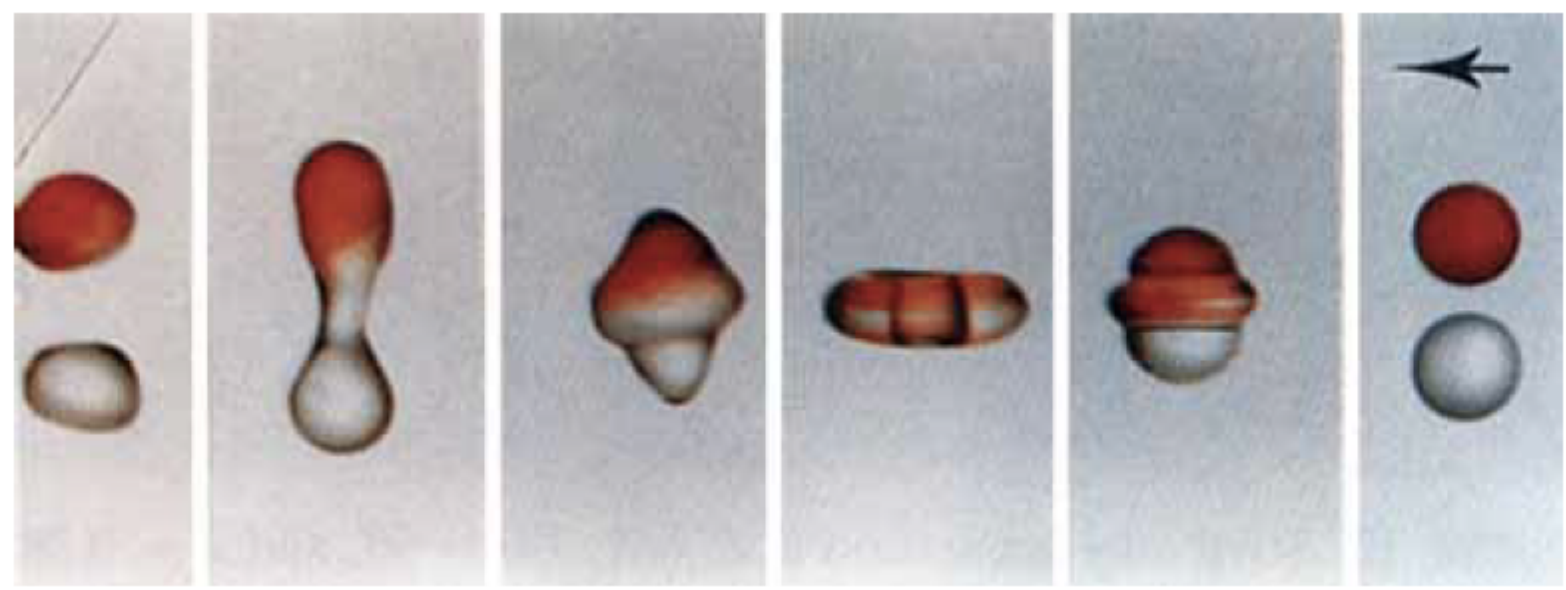

(a) $W e_{i m p}=23$
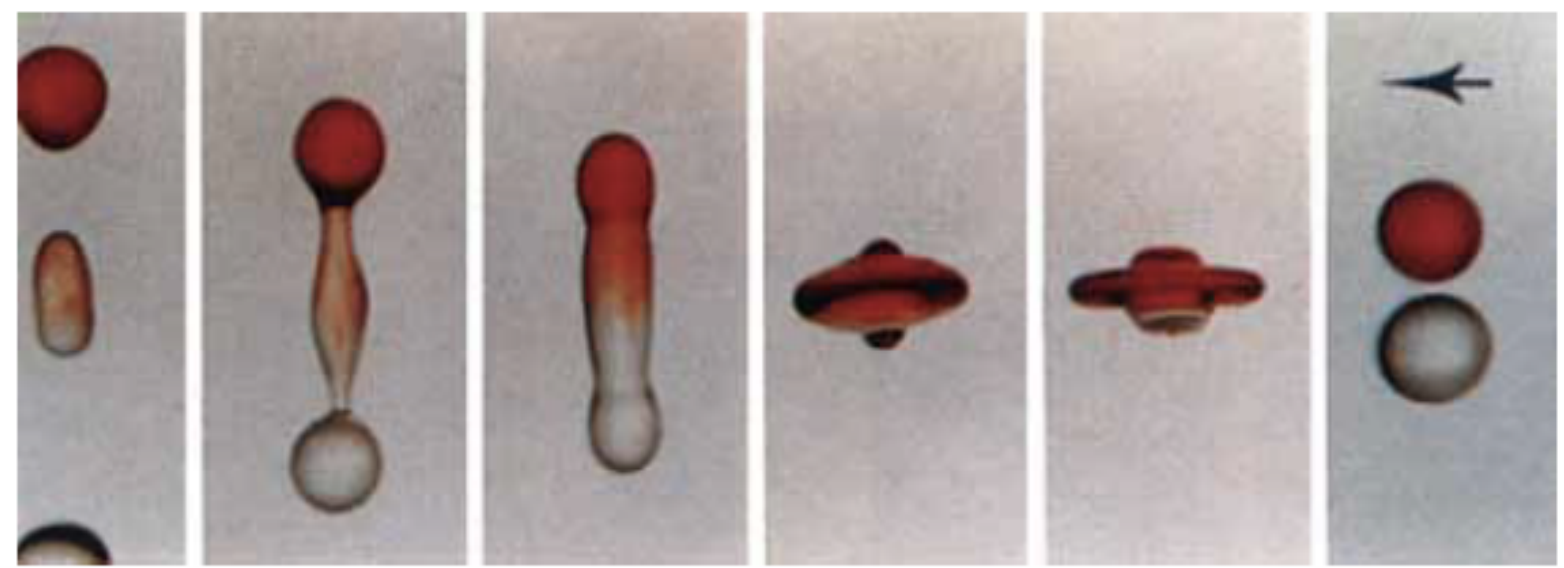

(b) $W e_{i m p}=40$

Figure 3. Experimental results for two weber of impact, from Ashgriz \& Poo [1].

The liquid fraction field for the case at $W e=50$ shown in Fig. 4 clearly highlights the diffusion induced by the DIM which tends to alter the regularity of the interface. Sharpening methods for 
compressible flows might be considered in a future work, following Shukla et al. [14].

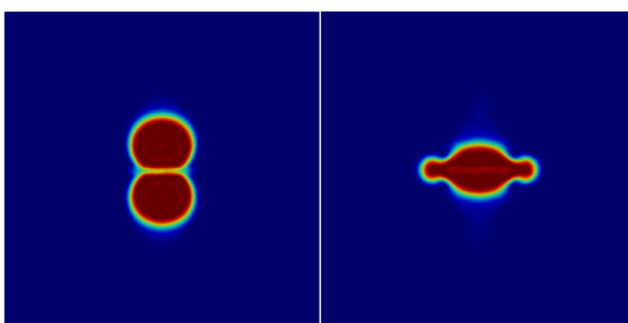

(a) $t=0.17 \mathrm{~ms}$ (b) $t=0.31 \mathrm{~ms}$

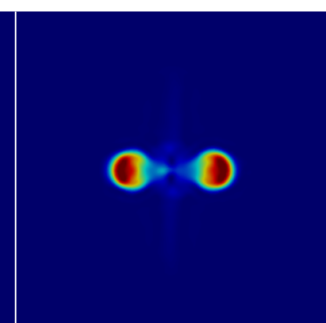

(c) $t=0.63 \mathrm{~ms}$

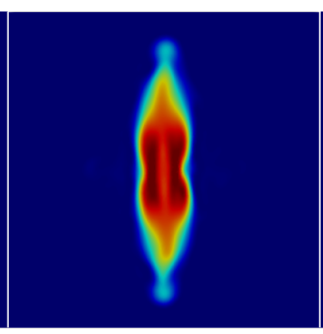

(d) $t=1.11 \mathrm{~ms}$



(e) $t=2.56 \mathrm{~ms}$

Figure 4. Field of $\alpha_{l}$, at different instants, $W e_{i m p}=40$.

\section{Application to a 2D turbulent jet.}

The method presented in this paper is finally applied to a 2D turbulent jet. The mixture used in this computation is single-component $\left(N_{2}\right)$ to focus on the capillary effects. The case is initially isobaric with the following density for liquid and gas: $\rho_{l}=800 \mathrm{~kg} \cdot \mathrm{m}^{-3}$ and $\rho_{g}=10 \mathrm{~kg} \cdot \mathrm{m}^{-3}$.

\section{Geometrical and Numerical set-up}

The mesh is a two dimensional triangular grid with 1 million cells and 40 cells in the injection diameter $D_{i n j}=0.1 \mathrm{~mm}$. Navier-Stokes equations are solved using a Two-Step Taylor Galerkin (TTG4A [12]) numerical scheme, 4th order in time, third order in space. A Dynamic Smagorinsky model (Germano et al. [10]) is used as a sub-grid scale model, together with a local artificial viscosity stabilization (Schmitt [13]).

The effect of surface tension on the resulting physics is evaluated by varying the Weber number defined in Eq. 17, changing $\sigma$ the surface tension coefficient, from $\sigma=0.05 \mathrm{~N} . \mathrm{m}^{-s}$ for $W e=50$ to $\sigma=0.005 N . m^{-1}$ for $W e=500$. The injection velocity $u_{i n j}=50 \mathrm{~m} . \mathrm{s}^{-1}$ and the liquid density $\rho_{l}=800 \mathrm{~kg} \cdot \mathrm{m}^{-1}$ are kept constant.

The liquid Reynolds number defined in Eq. 17 is equal to 40000 ensuring a turbulent liquid injection.

$$
W e_{j e t}=\frac{\rho_{l} u_{l}^{2} D_{i n j}}{\sigma} \quad R e_{l i q}=\frac{\rho_{l} u_{i n j} D_{i n j}}{\mu_{l}}
$$

While $W e_{j e t}=50$ characterizes a liquid flow strongly influenced by capillary effects, $W e_{j e t}=$ 500 characterizes a higher impact of the destabilizing inertial forces on the fragmentation of the liquid core.

\section{Results}

Instantaneous density fields are shown in Figs. 5-7 for $W e_{j e t}=50$ and $W e_{j e t}=500$. In both cases, the liquid jet smoothly penetrates into the gas, and start destabilizing quite early, before $0.2 \mathrm{~ms}$ (Fig. 5). At $0.4 \mathrm{~ms}$ both jets have reached the end of the computational domain ( Fig.6), and have become much turbulent. The liquid phase mixes with the gas in the form of thin ligaments, which then diffuse and tend to disappear at the latest times. The impact of the Weber number is clearly visible and as expected, with thinner ligaments at higher Weber numbers. These results highlight the diffusive behavior of DIM, which in this case prevents the ligaments to atomize into droplets. One strategy to overcome this issue is to artificially sharpen the interface ([14]). Another strategy is to force primary atomization before too much diffusion occurs, and transfer the atomized liquid mass to a Lagrange point source description. Both approaches are the subject of current works. 


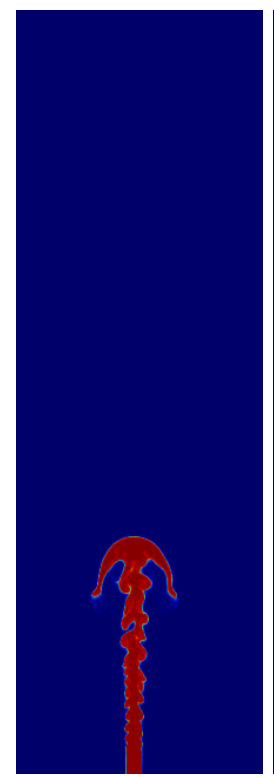

(a) $W e=50$

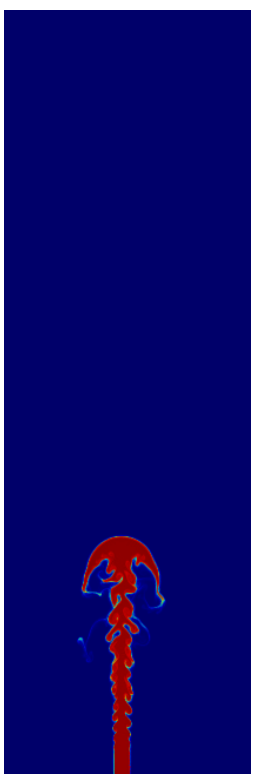

(b) $W e=500$

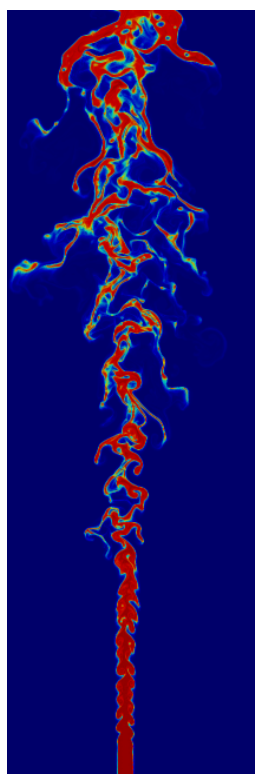

(a) $W e=50$

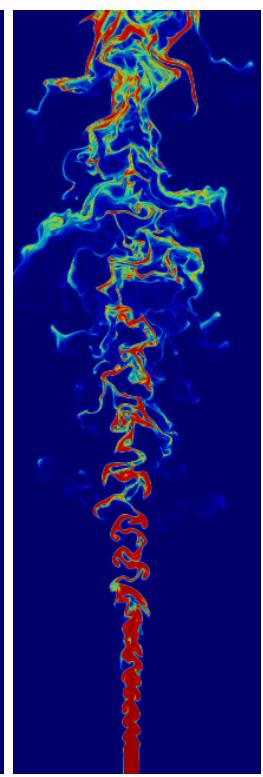

(b) $W e=500$

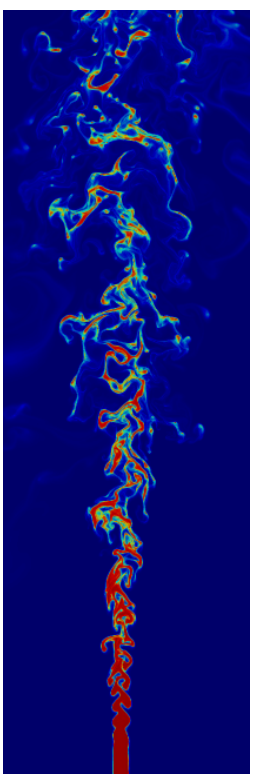

(a) $W e=50$

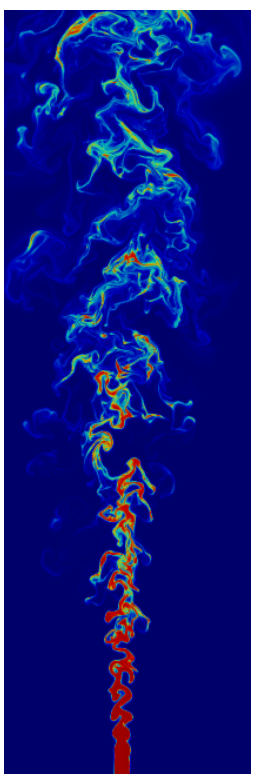

(b) $W e=500$

Figure 5. $t=0.2 \mathrm{~ms}$

Figure 7. $t=0.6 \mathrm{~ms}$

\section{Conclusions}

A Diffuse Interface method based on real gas thermodynamics and accounting for capillary effects is proposed in this paper, with the aim of simulating primary atomization of high-density ratio and multicomponent injection systems for industrial applications at reasonable computational cost. The considered real-gas thermodynamic closure relies on a cubic Peng-Robinson equation of state. The computation of the two-phase equilibrium in mono-component case relies on a tabulation of the saturation curves. The model is coupled with a surface tension description from Perigaud and Saurel [5] to address capillary effects. Validation is performed on three cases. First, Laplace equilibrium is recovered on a two-dimensional droplet. Then a three-dimensional binary collision of droplets is performed for two different Weber numbers. Qualitative results show that the trend of the fragmentation against the Weber number agrees with literature. This methodology is then applied to a 2D turbulent jet, representative of actual injection conditions. Liquid ligaments resulting from primary atomization seems recovered, and current work is now dedicated to the coupling with a Lagrange formalism to describe secondary atomization and predict the final spray.

\section{Acknowledgements}

This work has been founded by the European project SOPRANO. This work was performed using internal computation resources from CERFACS.

\section{References}

[1] Ashgriz, N. and Poo, J. Y., 1990, Coalescence and separation in binary collisions of liquid drops, J. Fluid Mech, vol 221, pp. 183-204.

[2] Pelletier, M., Schmitt, T., Ducruix. S, 2020, A multifluid Taylor-Galerkin methodology for the simulation of compressible multicomponent separate two-phase flows from subcritical to supercritical states, Computers \& Fluids, 206, pp. 104588.

[3] Baer, M. and Nunziato, J., Journal of Multiphase Flow 12, 861 - 889 (1986).

[4] Brackbill, J. U., Kothe, D. B., Zemach, C., 1992, A Continuum Method for Modeling Surface Tension, Journal of Computational Physics, pp. 335-354. 
[5] Perigaud, G., Saurel, R., 2005, A compressible flow model with capillary effects, Journal of Computational Physics, pp. 139-178.

[6] Colin, O., Rudgyard, M., 2000,Development of high-order Taylor-Galerkin schemes for unsteady calculations, Journal of Computational Physics, 162, pp. 338-371.

[7] Osher, S., Sethian, J. A., 1988, Fronts propagating with curvature dependant speed: Algorithm based on Hamilton-Jacobi formulations, Journal of Computational Physics, 79 (1), pp. 12-49.

[8] Hirt, C., W., Nichols, B. D., 1981, Volume of fluid (VOF) method for the dynamics of free boundaries, Journal of Computational Physics, 39(1), pp. 201-225.

[9] Poling, B. E., Prausnitz, J. M., 2001, The Properties of Gases and Liquids, McGraw-Hill.

[10] Germano, M., Piomello, U., Moin, P., Cabot, W., H., 1991, A dynamic subgrid-scale eddy viscosity model, Physics of Fluids A: Fluid Dynamics 3.

[11] Lefebvre, A. H., 1989, Atomization and Sprays.

[12] Schonfeld, T., \& Rudgyard, M. (1999). Steady and unsteady flows simulations using the hybrid flow solver avbp. AIAA Journal, 37, 1378-1385.

[13] Schmitt, T. 2020, Large-Eddy Simulations of the Mascotte Test Cases Operating at Supercritical Pressure, Flow, Turbulence and Combustion.

[14] Shukla, R. K., Pantano, C., Freund, J. B., 2010, An interface capturing method for the simulation of multi-phase compressible flows, Journal of Computational Physics, 229, pp. 7411-7439.

[15] Olsson, E., Kreiss, G., 2005, A conservative level set method for two phase flow, Journal of Computational Physics, 210, pp. 225-246.

[16] Pelletier, M., 2019, Diffuse interface models and adapted numerical schemes for the simulation of subcritical to supercritical flows, Ph.D thesis (CentraleSupélec), http://www.theses.fr/en/2019SACLC059

[17] Matheis, J., Hickel, S., 2018, Multi-component vapor-liquid equilibrium model for LES of high-pressure fuel injection and application to ECN Spray A, International Journal of Multiphase Flow, 99, pp. 294-311.

[18] Landau, L. D. \& Lifshitz, E. M. Fluid Mechanics Pergamon. New York, 1959, vol. 61.

[19] Pelletier, M. Diffuse interface models and adapted numerical schemes for the simulation of subcritical to supercritical flows. PhD, Paris Saclay 